\title{
EVALUACIÓN DEL PAICO Chenopodium ambrosioides Y CHOCHO Lupinus mutabilis SWEET COMO ANTIPARASITARIOS GASTROINTESTINALES EN BOVINOS JÓVENES
}

\author{
EVALUATION OF PAICO Chenopodium ambrosioides AND LUPINE Lupinus \\ mutabilis SWEET AS GASTROINTESTINAL ANTIPARASITIC IN YOUNG CATTLE
}

\author{
Francisco Clavijo López ${ }^{1, *}$, Víctor Barrera Mosquera ${ }^{1}$, Luis Rodríguez \\ Iturralde ${ }^{1}$, Jorge Mosquera Andrade ${ }^{2}$, Iván Yánez Ortiz ${ }^{1,2}$, Germán Godoy \\ Ortiz $^{1}$ y Jorge Grijalva Olmedo ${ }^{2}$
}

\footnotetext{
${ }^{1}$ Programa de Ganadería, Estación Experimental Santa Catalina, Instituto Nacional de Investigaciones Agropecuarias, Panamericana Sur Km. 1, Vía Tambillo, sector Cutuglagua. Telf. +593-2-3008750, Mejía, Ecuador.

${ }^{2}$ Facultad de Medicina Veterinaria y Zootecnia, Universidad Central del Ecuador, Jerónimo Leyton S/N y Gato Sobra, Telf. +593-2-2566160, Quito, Ecuador.
}

*Autor para correspondencia: fclavijolopez@gmail.com

Manuscrito recibido el 16 de enero de 2016. Aceptado, tras revisión, el 19 de septiembre de 2016.

\section{Resumen}

Las infestaciones parasitarias son una de las principales causas de enfermedades y pérdidas de la productividad en las explotaciones ganaderas en Ecuador, por lo que se hace necesario establecer mecanismos para reducirlas a través del uso de tratamientos alternativos a los químicos. En este estudio se evaluó el suministro del extracto acuoso del paico (Chenopodium ambrosioides) y del extracto fitoquímico de chocho (Lupinus mutábilis Sweet) en el control de parásitos gastrointestinales en bovinos. Se usaron diferentes dosis por kg de peso corporal con una y dos aplicaciones, frente a un testigo absoluto sin aplicación de producto y a un testigo convencional con aplicación de Fenbendazol; incluyó 10 tratamientos con 4 repeticiones representados por un animal como unidad experimental, asignados en un Diseño de Bloques Completos al Azar. Se evaluó el recuento e identificación de huevos de parásitos gastrointestinales preaplicación, la efectividad de los productos, la curva de reinfestación de los productos efectivos, la ganancia diaria de peso y los costos de cada tratamiento. Los resultados muestran que los géneros de parásitos encontrados fueron Trichostrongylus sp., Haemonchus sp. y Eimeria sp.; la efectividad hasta del 100\% en la reducción del número de huevos de parásitos gastrointestinales depende de la dosis y el número de aplicaciones; el período de reinfestación obedece a la residualidad del producto a partir del día 30 post-aplicación; y la ganancia diaria de peso no fue influenciada por la parasitosis de los animales. Se concluye que los productos orgánicos constituyen una estrategia biológica y económica factible para el control de parásitos gastrointestinales.

Palabras claves: extracto acuoso, reinfestación, residualidad, nemátodos, protozoarios. 


\begin{abstract}
Parasitic infestations are the main cause of productivity loss in the cattle industry in Ecuador. This makes necessary the establishment of mechanisms to help reduce these infestations through the use of alternative non-chemical treatments. This study evaluates the use of the aqueous extract from paico (Chenopodium ambrosioides) and the phytochemical extract from chocho (Lupinus mutábilis Sweet) to control gastrointestinal parasites in cattle. Different doses per $\mathrm{kg}$ of body weight were used with one and two applications, compared to a first control with no applications (absolute control) and a second control with Fenbendazol treatment (conventional control). Ten treatments were included with four repetitions, being one animal considered as an experimental unit. Treatments were assigned under a Randomized Complete Block Design. The variables evaluated were counting and identification of gastrointestinal parasites' eggs before each application, the effectiveness of the products, the curve of re-infestation of the effective products, daily weight gain, and the treatment cost. Results indicate that parasites found were Trichostrongylus sp., Haemonchus sp. y Eimeria sp. Also, an up to $100 \%$ of effectiveness in the reduction in the number of gastrointestinal parasites' eggs depends on the doses and the number of applications; the period of re-infestation obeys to the residually of the product from the 30th day post-application and on, and the daily weight gain was not impacted by the parasites in the animal. This study concludes that organic products are a feasible biological and economic strategy to control gastrointestinal parasites.
\end{abstract}

Keywords: aqueous extract, reinfestation, residual, nematodes, protozoa.

Forma sugerida de citar: Clavijo, F., et al. 2016. Evaluación del paico Chenopodium ambrosioides y chocho Lupinus mutabilis Sweet como antiparasitarios gastrointestinales en bovinos jóvenes. La Granja: Revista de Ciencias de la Vida. Vol. 24(2):95-110. ISSN: 1390-3799. 


\section{Introducción}

La explosión demográfica mundial exige una mayor eficiencia en la producción pecuaria para satisfacer la demanda de productos de origen animal; sin embargo, las pérdidas anuales producidas por parásitos internos son incalculables (Miño et al., 1998; Maya y Quijije, 2011). Una característica importante de los endoparásitos en los animales domésticos es que no son directamente apreciables y requieren de pruebas de laboratorio para demostrar y cuantificar su presencia, por lo que las infestaciones parasitarias son una de las principales causas de enfermedad y pérdida de la productividad en las explotaciones ganaderas de todo el mundo. Es precisamente en estos casos en los que es difícil determinar si los tratamientos antiparasitarios se justifican, es decir, si el beneficio económico que reportan compensa los gastos que conllevan y los problemas de contaminación y resistencia que ocasionan (Castro et al., 2011).

Este problema ha llevado a los criadores a usar empíricamente y en demasía los productos para contrarrestar la acción de los parásitos, generando resistencia a los productos químicos. Esto está preocupando mucho a los consumidores a nivel mundial, existiendo presión para adquirir alimentos inocuos, sin olvidar que los antiparasitarios producen también contaminación ambiental. En contra partida, se ha puesto en evidencia el conocimiento ancestral de comunidades indígenas sobre las propiedades y uso de muchos de los productos agrícolas propios de la serranía, lo que arrojaría ventajas como: sustentabilidad científica al conocimiento tradicional, solución a problemas sanitarios de animales, además de su fácil obtención (Torres, 2006).

Entre los beneficios que tienen estos medicamentos naturales se destaca la facilidad de administración, inocuidad, ningún efecto colateral indeseable en el animal y ausencia de residuos en la carne o leche; esto último, es una necesidad creciente entre los exportadores de productos convencionales (Muñoz, 2013). En este sentido, es posible aprovechar el efluente de la cocción del chocho Lupinus mutabilis Sweet, ya que por su alto contenido de alcaloides como la lupanina en un $46 \%$ y esparteína en un $14 \%$ de la materia sólida, puede ser utilizado en la desparasitación interna de los animales (Torres, 2006). De igual forma, se destaca el paico Chenopodium ambrosioides, ya que su aceite esencial contiene hasta el $90 \%$ de ascaridol, siendo este el componente activo responsable del efecto antiparasitario, según algunos autores como Trujillo y Madrigal (Trujillo y Madrigal, 2005).

Por los antecedentes mencionados que reflejan la influencia negativa de las parasitosis gastrointestinales en los bovinos jóvenes y los problemas que generan los antiparasitarios de origen químico, se efectuó la presente investigación, con el propósito de evaluar el efecto del suministro de esos productos sobre la carga parasitaria de los géneros de parásitos gastrointestinales presentes en bovinos jóvenes y sobre evaluar el período de reinfestación de parásitos gastrointestinales.

\section{Materiales y métodos}

\section{1 Área de estudio}

La ubicación geográfica y las características climáticas en donde se realizó la presente investigación se presentan en la Tabla 1.

\subsection{Método de investigación}

\subsubsection{Factores en estudio}

Los factores en estudio estuvieron constituidos por los productos naturales (extracto acuoso de paico y extracto fitoquímico de chocho), las dosis $\left(0.1 \mathrm{ml} \mathrm{kg}^{-1}\right.$ y $0.2 \mathrm{ml} \mathrm{kg}^{-1}$ de peso corporal de extracto acuoso de paico y $0.2 \mathrm{ml} \mathrm{kg}^{-1}$ y $0.4 \mathrm{ml} \mathrm{kg}^{-1}$ de peso corporal de extracto fitoquímico de chocho); la frecuencia de aplicación (una sola aplicación y dos aplicaciones); además de la aplicación de Fenbendazol a $0.05 \mathrm{ml} \mathrm{kg}^{-1}$ de peso corporal.

\subsubsection{Tratamientos}

Los tratamientos establecidos en la investigación se muestran en la Tabla 2.

\subsubsection{Diseño experimental}

Se utilizó un Diseño de Bloques Completos al Azar con 4 repeticiones. La unidad experimental estuvo constituida por un bovino joven entre 6 y 18 meses de edad, con parasitosis gastrointestinal. El bloqueo de los animales se realizó en base a su edad; es decir, en cada bloque se utilizó animales de diferentes edades. 
Tabla 1. Ubicación geográfica y características climáticas de la Estación Experimental Santa Catalina del INIAP, 2015.

\begin{tabular}{ll}
\hline Provincia: & Pichincha \\
Cantón: & Mejía \\
Parroquia: & Cutuglagua \\
Sitio: & Estación Experimental Santa Catalina \\
Altitud: & $3058 \mathrm{~m}$ \\
Latitud: & $00^{\circ} 22^{\prime} \mathrm{S}$ \\
Longitud: & $78^{\circ} 33^{\prime} \mathrm{W}$ \\
Temperatura anual: & $11.6^{\circ} \mathrm{C}$ \\
Precipitación media anual: & $1400 \mathrm{~mm}$ \\
Humedad relativa promedio: & $79 \%$ \\
Horas luz: & 12 a 13 horas \\
\hline Fuente: INAMHI, 2013. &
\end{tabular}

Tabla 2. Tratamientos para el estudio del paico y chocho como antiparasitarios en bovinos jóvenes. Estación Experimental Santa Catalina del INIAP, 2015.

\begin{tabular}{cl}
\hline Tratamiento & \multicolumn{1}{c}{ Descripción } \\
\hline T1 & Paico: $0.1 \mathrm{ml} \mathrm{kg}^{-1}$ de peso corporal + una sola aplicación (día 0) \\
T2 & Paico: $0.1 \mathrm{ml} \mathrm{kg}^{-1}$ de peso corporal + dos aplicaciones (día 0 y día 1) \\
T3 & Paico: $0.2 \mathrm{ml} \mathrm{kg}^{-1}$ de peso corporal + una sola aplicación (día 0) \\
T4 & Paico: $0.2 \mathrm{ml} \mathrm{kg}^{-1}$ de peso corporal + dos aplicaciones (día 0 y día 1) \\
T5 & Chocho: $0.2 \mathrm{ml} \mathrm{kg}^{-1}$ de peso corporal + una sola aplicación (día 0) \\
T6 & Chocho: $0.2 \mathrm{ml} \mathrm{kg}^{-1}$ de peso corporal + dos aplicaciones (día 0 y día 1) \\
T7 & Chocho: $0.4 \mathrm{ml} \mathrm{kg}^{-1}$ de peso corporal + una sola aplicación (día 0) \\
T8 & Chocho: $0.4 \mathrm{ml} \mathrm{kg}^{-1}$ de peso corporal + dos aplicaciones (día 0 y día 1) \\
T9 & Testigo absoluto (sin aplicación de producto) \\
T10 & Testigo convencional (Fenbendazol $0.05 \mathrm{ml} \mathrm{kg}$-1 de peso corporal) \\
\hline
\end{tabular}

Tabla 3. Esquema del Análisis de Varianza.

\begin{tabular}{lc}
\hline \multicolumn{1}{c}{ Fuente de variación } & Grados de libertad \\
\hline Total & 39 \\
Repeticiones & 3 \\
Tratamientos & 9 \\
Error experimental & 27 \\
\hline
\end{tabular}




\subsubsection{Análisis estadístico}

Se utilizó el análisis de varianza descrito en la Tabla 3. Los rangos de significación de las variables que resultaron significativas fueron establecidos mediante la prueba de Tukey al $5 \%$. Los datos obtenidos durante el proceso experimental fueron analizados con el software estadístico InfoStat versión 2015.

\subsubsection{Análisis económico}

Para el análisis económico se utilizó el método del Presupuesto Parcial propuesto por el CIMMYT (CIMMYT, 1988). Se recopilaron los costos y los beneficios de los tratamientos en estudio.

\subsubsection{Variables en estudio}

Recuento de huevos de parásitos gastrointestinales pre-aplicación

Se efectuó un examen coproparasitario antes de la aplicación de los productos, durante la evaluación de la efectividad de los mismos y durante el período de reinfestación, para lo cual se tomaron muestras de heces de cada unidad experimental y se trasladaron al Laboratorio de Diagnóstico Veterinario "VETELAB". La técnica utilizada fue la de recuento de huevos por gramo (HPG) en heces (Fiel et al., 2011; Sixtos, 2011).

\section{Identificación de los géneros de parásitos gastrointes-} tinales pre-aplicación

Se realizó conjuntamente con el recuento de huevos de parásitos gastrointestinales en las muestras de heces. La identificación de los géneros de parásitos gastrointestinales encontrados se la hizo según la guía propuesta por Díaz, (2013), en la que se describen los géneros de parásitos internos que pueden afectar a los animales domésticos, en este caso específico, a los bovinos.

\section{Determinación de la efectividad de los productos por género de parásito}

Se realizó un examen coproparasitario antes del tratamiento y a $\operatorname{los} 7,14$ y 21 días después de la aplicación de los productos (OIE, 2005). La efectividad o no de los productos aplicados se midió en porcentaje (\%). El porcentaje de reducción (eficacia) de la cantidad de huevos de parásitos se calculó a través de la siguiente fórmula (Power et al., 1982; OIE, 2005):

$$
P R=\frac{H P G_{\text {inicial }}-H P G_{\text {final }}}{H P G_{\text {inicial }}} \times 100,
$$

donde $P R=$ Porcentaje de reducción, $H P G_{\text {inicial }}=$ número de huevos por gramo de heces inicial y $H P G_{\text {final }}=$ número de huevos por gramo de heces final.

La efectividad fue evaluada en base a: Altamente efectivo $\geq 98 \%$, Efectivo $=90-98 \%$, Ayuda en el control $=80-89 \%$, e Insuficientemente activo $\leq 80 \%$ (no registrable) (OIE, 2005).

\section{Determinación de la curva de reinfestación de los tra- tamientos efectivos}

Para la evaluación de la curva de reinfestación se realizó un examen coproparasitario a los 30,60 y 90 días después de ser determinada la efectividad de los productos aplicados. La curva de reinfestación relacionó el tiempo, es decir, los días después de la desparasitación con los productos aplicados y el número de huevos de parásitos encontrados en ese tiempo de los respectivos géneros identificados.

\section{Determinación de peso, incremento de peso y ganan- cia diaria de peso}

Para la determinación del peso, incremento de peso y ganancia diaria de peso (GDP), se realizó el pesaje de cada unidad experimental mediante el uso de una cinta zoométrica, a los 0, 7, 14 y 21 días, a partir de la aplicación de los productos. La ganancia diaria de peso (GDP) se midió en gramos, aplicando la siguiente fórmula:

$$
G D P=\frac{\text { Peso final }- \text { Peso inicial }}{\text { Duración del periodo }} .
$$

\subsubsection{Manejo del experimento}

Se seleccionaron animales entre 6 a 18 meses de edad de acuerdo a los registros llevados en el Programa de Ganadería de la Estación Experimental Santa Catalina del INIAP. El manejo de estos animales fue utilizando un sistema de pastoreo rotativo en franjas, con potreros constituidos por una mezcla forrajera de kikuyo Pennisetum clandestinum, ray 
grass perenne Lolium perenne, ray grass anual Lolium multiflorum, pasto azul Dactylis glomerata, trébol blanco Trifolium repens y trébol rojo Trifolium pratense; $2 \mathrm{~kg}$ de balanceado animal ${ }^{-1}$ día $^{-1}$, agua a voluntad, además de una suplementación con sal mineral, en una proporción de $50 \mathrm{~g}_{\text {animal }}{ }^{-1}$ día $^{-1}$.

Para el análisis inicial del parasitismo gastrointestinal, se realizó un examen coproparasitario a cada uno de los animales en estudio, con el propósito de identificar los géneros de parásitos gastrointestinales presentes y conocer el grado de infestación. Para la toma de muestras de heces se siguieron las técnicas propuestas por Thienpont, (1989) y Fiel et al., (2011), y para el recuento de huevos de parásitos gastrointestinales, se empleó la técnica de Mc Máster (Sixtos, 2011).

Después de obtener los resultados de los coproparasitarios iniciales de cada unidad experimental, se procedió a desparasitar a los animales, para lo que se aplicó BENDACURß, que es un antihelmíntico de amplio espectro contra parásitos gastrointestinales y pulmonares a base de Fenbendazol, en dosis de $1 \mathrm{ml}$ por cada $20 \mathrm{~kg}$ de peso corporal y por vía oral (James Brown Pharma, 2013). Esto se realizó para garantizar que todos los animales estén libres y no vayan a presentar parásitos gastrointestinales en su organismo.

Una vez que los animales en estudio se encontraron libres de parásitos gastrointestinales, se procedió a parasitarlos, para lo que fueron mantenidos a pastoreo por un período de 21 días, en potreros cerrados (se dio preferencia a un potrero que contuviera anteriormente a animales adultos y no tratados), en la búsqueda de que se produzca una contaminación natural de los animales (OIE, 2005). Adicionalmente, se realizó una infestación artificial o inducida a los animales en estudio, mediante la aplicación de un inóculo con larvas infestantes (L3) en dosis de $5 \mathrm{ml}$, para lo que se tomó una muestra de heces de un animal parasitado y se cultivó dicha muestra por 21 días (Prada, 2008; Soca et al., 2005; Angulo, 2005). Esto se realizó para que todas las unidades experimentales tengan una similitud, tanto en carga parasitaria como en los diferentes géneros de parásitos gastrointestinales.

Después del período de infestación natural (21 días) y de la aplicación del inóculo con las larvas infestantes (L3), se hizo un análisis pre-tratamiento del parasitismo gastrointestinal, para lo que se realizaron exámenes coproparasitarios, aplicando las mismas técnicas y procedimientos ya descritos, pa- ra determinar el grado de parasitación y los géneros de nemátodos presentes en cada unidad experimental. De acuerdo a los resultados obtenidos, los animales fueron distribuidos al azar en 10 grupos con 4 repeticiones.

Para la preparación del extracto acuoso de paico, que fue administrado como desparasitante, se debió extraer el zumo del paico, para lo que se tomó $1 \mathrm{~kg}$ de las hojas de la planta en un mortero y se las trituró; se añadió 2 litros de agua; luego se filtró y se utilizó (Supe, 2008).

Para la preparación del extracto fitoquímico de chocho, que fue administrado como desparasitante, se utilizó el siguiente proceso: se pesó $1 \mathrm{~kg}$ de chocho maduro y seco, se lo colocó en un recipiente plástico con 4 litros de agua potable para iniciar el tiempo de remojo (12 horas); luego se procedió al cocido en una olla por 40 minutos para obtener el extracto del chocho que después se lo tamizó y enfrió (Torres, 2006).

La aplicación de los productos naturales se realizó por vía oral en todos los tratamientos. Según Finkeros (Finkeros, 2012), la dosis del extracto acuoso de paico, que se usa como antiparasitario gastrointestinal en bovinos, es de $0.1 \mathrm{ml} \mathrm{kg}^{-1}$ de peso corporal; mientras que, según Torres, (2006), la dosis del extracto fitoquímico de chocho, que se usa como antiparasitario gastrointestinal en bovinos, es de $0.2 \mathrm{ml} \mathrm{kg}^{-1}$ de peso corporal.

Para el tratamiento convencional se aplicó

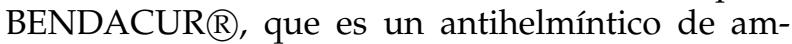
plio espectro contra parásitos gastrointestinales y pulmonares a base de Fenbendazol, en dosis de $1 \mathrm{ml}$ por cada $20 \mathrm{~kg}$ de peso corporal y por vía oral (James Brown Pharma, 2013). Para el tratamiento testigo absoluto no se aplicó ningún producto.

Luego de la administración de los productos a los animales en estudio, de acuerdo al respectivo tratamiento, se procedió a realizar exámenes coproparasitarios a $\operatorname{los} 7,14$ y 21 días. Esto se realizó para determinar la efectividad o no de estos productos frente a los parásitos gastrointestinales encontrados e identificados inicialmente.

Luego de ser determinada la efectividad de los productos aplicados, se estableció la curva de reinfestación en los tratamientos que fueron efectivos como antiparasitarios, para lo cual se realizaron exámenes coproparasitarios a los 30,60 y 90 días. Esto se lo hizo para establecer el tiempo en que los animales vuelven a adquirir el parasitismo gastrointestinal, así como para determinar el tiempo du- 
rante el cual cada uno de los productos protege al animal de dicho parasitismo.

\section{Resultados y discusión}

\subsection{Recuento e identificación de huevos de parásitos gastrointestinales pre- tratamiento}

Previo a la aplicación de los productos se observó que todos los tratamientos dieran positivo a parasitosis gastrointestinal; se obtuvo una infestación de nemátodos promedio de $150 \mathrm{HPG}$, una mediana de 100 HPG y un rango de 50 a 600 HPG de heces; mientras que en protozoarios, se obtuvo una infestación promedio de 180 OPG, una mediana de 150 OPG y un rango de 50 a 600 OPG de heces. La variabilidad en la distribución de las cargas parasitarias en el seno de la población de hospedadores, reflejan la heterogeneidad en la predisposición de algunos individuos para infestarse, aun estando bajo las mismas condiciones de exposición a las larvas (L3) infestantes, por lo que la susceptibilidad de los hospedadores se traduce en diferencias, tanto del porcentaje de animales infestados como en la distribución de las cargas parasitarias en los animales. Estas diferencias en la carga parasitaria tienen una base genética, pero en interacción con otros factores de muy diversa índole, como los nutricionales, estado fisiológico, sexo, edad, condiciones medioambientales y condiciones de estrés, entre otros (Morales et al., 2003).

Al realizar la identificación de los parásitos gastrointestinales presentes, previo a la aplicación de los productos, se encontraron huevos de nemátodos del género Trichostrongylus sp. y Haemonchus sp. De los 40 animales evaluados, 12 presentaron únicamente la infestación por Trichostrongylus sp., lo que corresponde al $30 \%$; para Haemonchus sp. se infestaron 8 animales que corresponden al 20\%; y los 20 animales restantes se infestaron con los dos géneros, lo que corresponde al 50\%. Además, se identificaron protozoarios del género Eimeria sp. con una infestación del 100\% de los animales. Según Morales et al. (Morales et al., 2003), el recuento de huevos por gramo de heces en bovinos menores de un año y en bovinos jóvenes de hasta 18 meses, hace referencia a los géneros Haemonchus sp., Trichostrongylus sp. y Cooperia sp., como los que más contribuyen con la producción de huevos por gramo de heces.

\subsection{Efectividad de los productos sobre la carga parasitaria de nemátodos del gé- nero Trichostrongylus sp.}

La Tabla 4 muestra los promedios y la prueba de Tukey al $5 \%$ para la variable efectividad de los productos sobre la carga parasitaria de nemátodos del género Trichostrongylus sp. a los 7 días postaplicación; se reportan dos rangos de significación, encontrándose solo en el rango $a$ al tratamiento T9 con $0 \%$ de efectividad, en los rangos $a b$ a los tratamientos T3, T4, T5, T6, T7 y T8 con porcentajes de efectividad entre $50 \%$ y $75 \%$, y solo en el rango $b$ a los tratamientos T1, T2 y T10 con porcentajes de efectividad entre $87.50 \%$ y $100 \%$. Según OIE OIE, (2005), un porcentaje de efectividad entre el 80 al $89 \%$ ayuda al control, mientras que uno igual o superior al $90 \%$ es efectivo; por lo tanto, los tratamientos considerados efectivos son los tratamientos T10 y T1 con el $100 \%$ de efectividad y el tratamiento T2 con el $87.50 \%$ de efectividad sería calificado como una ayuda en el control.

Los promedios y la prueba de Tukey al $5 \%$ para la variable efectividad de los productos sobre la carga parasitaria de nemátodos del género Trichostrongylus sp. a los 14 días post-aplicación (Tabla 4), muestran dos rangos de significación, reportándose solo en el rango $a$ al tratamiento T9 con $0 \%$ de efectividad, en los rangos $a b$ a los tratamientos T4, T5, T6, T7 y T8 con porcentajes de efectividad entre $37.50 \%$ y $75 \%$, y solo en el rango $b$ a los tratamientos T1, T2, T3 y T10 con porcentajes de efectividad entre $79.17 \%$ y $100 \%$. Los tratamientos considerados efectivos son los tratamientos T10 y T1 con el 100\% de efectividad y el tratamiento T3 con el $83.33 \%$ de efectividad sería calificado como una ayuda en el control.

Para la variable efectividad de los productos sobre la carga parasitaria de nemátodos del género Trichostrongylus sp. a los 21 días post-aplicación, los promedios y prueba de Tukey al 5\% (Tabla 4), reportan dos rangos de significación, encontrándose solo en el rango $a$ al tratamiento T9 con $0 \%$ de efectividad, en los rangos $a b$ a los tratamientos T4, T5, T6 y T7 con porcentajes de efectividad entre $50 \%$ y $70 \%$, y solo en el rango $b$ a los tratamientos T1, T2, T3, T8 y T10 con porcentajes de efectividad entre $77.08 \%$ y $100 \%$; por lo tanto, los tratamientos considerados efectivos son los tratamientos T2, T8 y T10 con $100 \%$ de efectividad y el tratamiento T3 con $91.67 \%$ de efectividad. 
Tabla 4. Promedios, error estándar y prueba de Tukey al 5\% para la variable efectividad de los productos sobre la carga parasitaria de nemátodos del género Trichostrongylus sp., durante el periodo de evaluación. Estación Experimental Santa Catalina del INIAP, 2015 .

\begin{tabular}{|c|c|c|c|}
\hline \multirow[t]{2}{*}{ Tratamientos en estudio } & \multicolumn{3}{|c|}{$\begin{array}{c}\text { Porcentaje de reducción de HPG } \\
\text { de Trichostrongylus sp. }\end{array}$} \\
\hline & 7 días & 14 días & 21 días \\
\hline T1= Paico: $0.1 \mathrm{ml} \mathrm{kg}^{-1}$ de peso corporal + una sola aplicación (día 0) & $100.00 \mathrm{~b}$ & $100.00 \mathrm{~b}$ & $77.08 b$ \\
\hline $\mathrm{T} 2=$ Paico: $0.1 \mathrm{ml} \mathrm{kg}^{-1}$ de peso corporal + dos aplicaciones (día 0 y día 1 ) & $87.50 b$ & $79.17 b$ & $100.00 b$ \\
\hline T3= Paico: $0.2 \mathrm{ml} \mathrm{kg}^{-1}$ de peso corporal + una sola aplicación (día 0) & $70.83 a b$ & $83.33 b$ & $91.67 b$ \\
\hline T4= Paico: $0.2 \mathrm{ml} \mathrm{kg}^{-1}$ de peso corporal + dos aplicaciones (día 0 y día 1 ) & $68.18 a b$ & $70.46 b$ & $70.00 a b$ \\
\hline T5= Chocho: $0.2 \mathrm{ml} \mathrm{kg}^{-1}$ de peso corporal + una sola aplicación (día 0) & $50.00 a b$ & $37.50 a b$ & $50.00 a b$ \\
\hline T6= Chocho: $0.2 \mathrm{ml} \mathrm{kg}^{-1}$ de peso corporal + dos aplicaciones (día 0 y día 1 ) & $75.00 a b$ & $75.00 a b$ & $62.50 a b$ \\
\hline T7 = Chocho: $0.4 \mathrm{ml} \mathrm{kg}^{-1}$ de peso corporal + una sola aplicación (día 0) & $75.00 a b$ & $75.00 a b$ & $62.50 a b$ \\
\hline $\mathrm{T} 8=$ Chocho: $0.4 \mathrm{ml} \mathrm{kg}^{-1}$ de peso corporal + dos aplicaciones (día 0 y día 1 ) & $75.00 a b$ & $75.00 a b$ & $100.00 b$ \\
\hline T9= Testigo absoluto (sin aplicación de producto) & $0.00 a$ & $0.00 a$ & $0.00 a$ \\
\hline $\mathrm{T} 10=$ Testigo convencional (Fenbendazol $0.05 \mathrm{ml} \mathrm{kg}^{-1}$ de peso corporal) & $100.00 b$ & $100.00 b$ & $100.00 \mathrm{~b}$ \\
\hline Error estándar de la media $\left(s_{\bar{x}}\right)$ & 19.44 & 17.40 & 16.50 \\
\hline
\end{tabular}

Los resultados de efectividad obtenidos de los productos aplicados sobre la carga parasitaria del género Trichostrongylus sp., muestran que la aplicación de Fenbendazol fue efectiva en todo el período de evaluación, lo cual corrobora lo dicho por Fierro, (2010), "el control parasitario del Trichostrongylus sp. fue efectivo hasta los 20 días con la aplicación de Ivermectina", y ratifica la eficiencia antiparasitaria del Fenbendazol y otros antihelmínticos (Benzimidazoles e Avermectinas), tal como lo han demostrado Morales et al., (2003), considerándose un antihelmíntico de amplio espectro con acción vermicida, larvicida y ovicida sobre parásitos de diferentes géneros. Morales et al., (2006), determinaron una elevada eficiencia en los antihelmínticos utilizados en finca (Febendazole y Sulfoxido de Albendazole); sin embargo, su acción persiste durante poco tiempo y su uso constante puede llevar a problemas de resistencia (Anziani y Fiel, 2004).

Los extractos evaluados presentaron un control variable sobre la carga parasitaria de este género, siendo los más efectivos el T1 con un promedio del $92.36 \%$ de efectividad y el T2, con un promedio del $88.89 \%$ de efectividad durante todo el período de evaluación, por lo que se considera que estos trata- mientos de origen natural presentan respuestas favorables para el control de este parásito, ya que tienen una efectividad promedio superior al $80 \%$.

La efectividad del extracto acuoso de paico se debe al hecho de poseer ascaridol, que es un antihelmíntico natural que altera el metabolismo e inhibe la enzima fumarato reductasa de las mitocondrias, enzima que convierte fumarato a succinato y es importante en el metabolismo microbiano para la respiración anaeróbica, la disminución del transporte de glucosa o el desacoplamiento de la fosforilación oxidativa, que es un proceso metabólico que utiliza energía liberada por la oxidación de nutrientes para producir adenosín trifosfato (ATP), destruyendo al parásito (Jurado et al., 2007; Chacón et al., 2009). Este efecto es ratificado por Álvarez et al., (2011), quienes al utilizar el extracto acuoso de paico como tratamiento alternativo antiparasitario en los gallos de pelea, comprobó su eficacia en la disminución de huevos de nematodos; esto, debido al efecto antinematocida del ascaridol, el cual produce un efecto paralizante y narcótico sobre el parásito, haciendo que se desprenda del tejido intestinal al cual están adheridos y sea eliminado a través de la materia fecal. En otro estudio realizado por Estrada-Celi et al., 
(2012), el paico presenta una efectividad del 100\% en el control de helmintos en especímenes silvestres mantenidos en cautiverio.

\subsection{Efectividad de los productos sobre la carga parasitaria de nemátodos del gé- nero Haemonchus sp.}

La prueba de Tukey al 5\% para la variable efectividad de los productos sobre la carga parasitaria de nemátodos del género Haemonchus sp. a los 7 días post-aplicación (Tabla 5), muestra dos rangos de significación, reportándose solo en el rango $a$ al tratamiento $\mathrm{T} 9$ con $0 \%$ de efectividad, en los rangos $a b$ a los tratamientos T1, T2, T3, T4, T5, T7 y T8 con porcentajes de efectividad entre $25 \%$ y $87.50 \%$, y solo en el rango $b$ a los tratamientos T6 y T10 con porcentajes de efectividad entre $95.83 \%$ y $100 \%$.

Si bien no se reportaron diferencias estadísticas entre los promedios de los tratamientos a los $14 \mathrm{y}$ 21 días post-aplicación, los tratamientos T10 y el T5 presentaron los mayores porcentajes de efectividad con el 100\%; caso contrario aconteció con el T9 que presentó un $25 \%$ de efectividad a los 14 y 21 días post-aplicación. Este incremento en el porcentaje de efectividad puede deberse a las defensas propias del animal e influencias del medio en los parásitos, así como al buen control de parásitos en los potreros en donde se realizó la investigación.

Los resultados de efectividad obtenidos de los productos aplicados sobre la carga parasitaria del género Haemonchus sp., muestran que la aplicación de Fenbendazol obtuvo el $100 \%$ de efectividad en todo el período de evaluación; esto corrobora lo dicho por González et al., (2003), "el control parasitario del género Haemonchus sp. es efectivo hasta los 30 días con la aplicación de Levamisol".

La respuesta de la aplicación de los extractos evaluados sobre Haemonchus sp. fue variable, siendo los más efectivos los tratamientos $\mathrm{T} 5$ con un promedio del $95.83 \%$ de efectividad y el T6 con un promedio del $81.94 \%$ de efectividad durante todo el período de evaluación, por lo que se considera que estos tratamientos de origen natural presentan respuestas favorables para el control de este parásito, ya que tienen una efectividad promedio superior al $80 \%$.

Tabla 5. Promedios, error estándar y prueba de Tukey al $5 \%$ para la variable efectividad de los productos sobre la carga parasitaria de nemátodos del género Haemonchus sp., durante el periodo de evaluación. Estación Experimental Santa Catalina del INIAP, 2015.

\begin{tabular}{|c|c|c|c|}
\hline \multirow[t]{2}{*}{ Tratamientos en estudio } & \multicolumn{3}{|c|}{$\begin{array}{c}\text { Porcentaje de reducción de HPG } \\
\text { de Haemonchus sp. }\end{array}$} \\
\hline & 7 días & 14 días & 21 días \\
\hline $\mathrm{T} 1=$ Paico: $0.1 \mathrm{ml} \mathrm{kg}^{-1}$ de peso corporal + una sola aplicación (día 0) & $75.00 a b$ & $68.75 a$ & $68.75 a$ \\
\hline $\mathrm{T} 2=$ Paico: $0.1 \mathrm{ml} \mathrm{kg}^{-1}$ de peso corporal + dos aplicaciones (día 0 y día 1 ) & $50.00 a b$ & $50.00 a$ & $50.00 a$ \\
\hline T3= Paico: $0.2 \mathrm{ml} \mathrm{kg}^{-1}$ de peso corporal + una sola aplicación (día 0) & $25.00 a b$ & $50.00 a$ & $25.00 a$ \\
\hline T4= Paico: $0.2 \mathrm{ml} \mathrm{kg}^{-1}$ de peso corporal + dos aplicaciones (día 0 y día 1 ) & $67.50 a b$ & $65.00 a$ & $75.00 a$ \\
\hline T5= Chocho: $0.2 \mathrm{ml} \mathrm{kg}^{-1}$ de peso corporal + una sola aplicación (día 0) & $87.50 a b$ & $100.00 a$ & $100.00 a$ \\
\hline T6= Chocho: $0.2 \mathrm{ml} \mathrm{kg}^{-1}$ de peso corporal + dos aplicaciones (día 0 y día 1 ) & $95.83 b$ & $100.00 a$ & $100.00 a$ \\
\hline T7= Chocho: $0.4 \mathrm{ml} \mathrm{kg}^{-1}$ de peso corporal + una sola aplicación (día 0) & $50.00 a b$ & $75.00 a$ & $50.00 a$ \\
\hline $\mathrm{T} 8=$ Chocho: $0.4 \mathrm{ml} \mathrm{kg}^{-1}$ de peso corporal + dos aplicaciones (día 0 y día 1 ) & $37.50 a b$ & $37.50 a$ & $50.00 a$ \\
\hline T9= Testigo absoluto (sin aplicación de producto) & $0.00 a$ & $25.00 a$ & $25.00 a$ \\
\hline $\mathrm{T} 10=$ Testigo convencional (Fenbendazol $0.05 \mathrm{ml} \mathrm{kg}^{-1}$ de peso corporal) & $100.00 b$ & $100.00 a$ & $100.00 a$ \\
\hline Error estándar de la media $\left(s_{\bar{x}}\right)$ & 19.49 & 19.85 & 21.06 \\
\hline
\end{tabular}

Letras diferentes en la misma columna indican diferencias estadísticas significativas $(\mathrm{P} \leq 0.05)$. 
El extracto fitoquímico del chocho presentó una buena efectividad para el control de nemátodos gastrointestinales, ya que durante la evaluación se registraron disminuciones en la carga parasitaria, propiciando un corte del ciclo de estos parásitos, situación que apunta a que los alcaloides presentes en el extracto fitoquímico del chocho, como son la lupanina y esparteína, tienen un buen efecto antiparasitario para controlar los parásitos intestinales de los animales (Gross, 1992; Torres, 2006), lo que ratifica los resultados obtenidos, ya que por el efecto demostrado se registra una disminución de estos parásitos a partir de los 7 días después de la aplicación.

\subsection{Efectividad de los productos sobre la carga parasitaria de protozoarios del género Eimeria sp.}

En la Tabla 6 se reportan los promedios de las variables efectividad de los productos sobre la carga parasitaria de protozoarios del género Eimeria sp. a los 7, 14 y 21 días post-aplicación. A pesar que no se reportaron diferencias estadísticas entre los promedios de los tratamientos a los 7, 14 y 21 días postaplicación, el tratamiento T2 presentó los mayores porcentajes de efectividad de $80 \%, 85 \%$ y $87.50 \%$, a los 7, 14 y 21 días post-aplicación, respectivamente.

Ningún tratamiento fue completamente efectivo para el control Eimeria sp., pero los tratamientos que redujeron la carga parasitaria de protozoarios del género Eimeria sp. fueron el T2 y el T5. Estos resultados dan a relucir que el ascaridol contenido en el extracto acuoso de paico al ser un antihelmíntico natural, posee efectos negativos sobre el crecimiento de este género de parásitos (Jurado et al., 2007; Chacón et al., 2009). De igual manera se deduce que los alcaloides lupanina y esparteína presentes en el extracto fitoquímico del chocho, tienen un buen efecto antihelmíntico (Gross, 1992; Torres, 2006).

\subsection{Curva de reinfestación de huevos de ne- mátodos gastrointestinales a partir de los 21 días post-aplicación en los trata- mientos efectivos}

Los tratamientos efectivos ( $>90 \%$ ) hasta los 21 días post-aplicación fueron el T1, T2, T5, T6 y T10. Existe variabilidad en la reinfestación de huevos de nemátodos gastrointestinales de los géneros Trichos- trongylus sp. y Haemonchus sp. durante la evaluación a los 30,60, y 90 días en los tratamientos evaluados (Figura 1); esto se atribuye a que la reinfestación de nemátodos es variable dentro de las diferentes especies de parásitos así como en función de ciertas características individuales del hospedador, como la edad y el estado inmunológico. Otro aspecto a considerar en este caso se refiere a la presencia de individuos susceptibles a la reinfección como consecuencia de la constante exposición a los parásitos y la corta persistencia del producto administrado en forma convencional (FAO, 2003).

La forma de transmisión de los parásitos a los bovinos posiblemente se debió al suministro de forraje contaminado, ya que según Torres y Borchert (Torres, 2006; Borchert, 1993), el contagio tiene lugar por ingestión de forraje proveniente de prados infestados, que están determinados por lo general de factores biológicos, hidrológicos y climáticos de la zona, ya que el contagio de los animales resulta tanto más intenso cuando mejores sean las condiciones para el desarrollo de los huevos.

Los resultados obtenidos se asemejan con lo descrito por Fierro, (2010), quien menciona que al aplicar el extracto fitoquímico de chocho a los 35 días se reduce la carga parasitaria en los camélidos tratados, pero se incrementa nuevamente a los 50 días. En tanto que con la aplicación del extracto acuoso de paico se obtiene un incremento en la carga parasitaria a partir del día 30 de la aplicación. Según Valencia, (2014), los antiparasitarios químicos, como el Fenbendazol, son productos de amplio espectro que afectan a nemátodos gastrointestinales y pulmonares, además de que poseen efecto larvicida, por lo que no es necesario repetir la desparasitación entre los 21 y 28 días de la primera aplicación. Estos productos causan degeneración de los microtúbulos citoplasmáticos e inhiben el transporte de la glucosa interfiriendo con la producción de energía en el interior del parásito; por lo que, se recomienda hacer intervalos de desparasitación de 3 a 4 meses en los animales jóvenes, dependiendo del desafío parasitario.

Cordero et al., (2005), mencionan que el antiparasitario más eficiente elimina la totalidad de los parásitos que se hallan dentro del animal, pero un mes después de nuevo habrá parásitos adultos en el tubo digestivo debido a la ingestión de los hospedadores intermediarios con las pasturas. El mismo fenómeno se observó en el presente estudio, ya que hubo una reducción en la carga parasitaria a 
los días 7, 14 y 21 post-aplicación, pero en el día 30 existió presencia de huevos de nemátodos gastrointestinales. El manejo antiparasitario eficiente debe pensarse no solo para eliminar la carga de parási- tos en los animales puntualmente sino también para disminuir la reinfestación (Caracostántogolo et al., 2005).

Tabla 6. Promedios y error estándar para la efectividad de los productos sobre la carga parasitaria de protozoarios del género Eimeria sp., durante el periodo de evaluación. Estación Experimental Santa Catalina del INIAP, 2015.

\begin{tabular}{|c|c|c|c|}
\hline \multirow[t]{2}{*}{ Tratamientos en estudio } & \multicolumn{3}{|c|}{$\begin{array}{c}\text { Porcentaje de reducción de HPG } \\
\text { de Eimeria } \text { sp. }\end{array}$} \\
\hline & 7 días & 14 días & 21 días \\
\hline T1= Paico: $0.1 \mathrm{ml} \mathrm{kg}^{-1}$ de peso corporal + una sola aplicación (día 0 ) & $43.75 a$ & $68.75 a$ & $75.00 a$ \\
\hline $\mathrm{T} 2=$ Paico: $0.1 \mathrm{ml} \mathrm{kg}^{-1}$ de peso corporal + dos aplicaciones (día 0 y día 1 ) & $80.00 a$ & $85.00 a$ & $87.50 a$ \\
\hline T3= Paico: $0.2 \mathrm{ml} \mathrm{kg}^{-1}$ de peso corporal + una sola aplicación (día 0) & $36.67 a$ & $77.50 a$ & $85.00 a$ \\
\hline T4= Paico: $0.2 \mathrm{ml} \mathrm{kg}^{-1}$ de peso corporal + dos aplicaciones (día 0 y día 1 ) & $61.11 a$ & $61.11 a$ & $72.92 a$ \\
\hline T5= Chocho: $0.2 \mathrm{ml} \mathrm{kg}^{-1}$ de peso corporal + una sola aplicación (día 0) & $41.67 a$ & $83.33 a$ & $83.33 a$ \\
\hline T6= Chocho: $0.2 \mathrm{ml} \mathrm{kg}^{-1}$ de peso corporal + dos aplicaciones (día 0 y día 1 ) & $31.25 a$ & $68.75 a$ & $25.00 a$ \\
\hline T7= Chocho: $0.4 \mathrm{ml} \mathrm{kg}^{-1}$ de peso corporal + una sola aplicación (día 0) & $44.17 a$ & $70.00 a$ & $50.00 a$ \\
\hline $\mathrm{T} 8=$ Chocho: $0.4 \mathrm{ml} \mathrm{kg}^{-1}$ de peso corporal + dos aplicaciones (día 0 y día 1 ) & $37.50 a$ & $37.50 a$ & $50.00 a$ \\
\hline T9= Testigo absoluto (sin aplicación de producto) & $25.00 a$ & $50.00 a$ & $43.75 a$ \\
\hline $\mathrm{T} 10=$ Testigo convencional (Fenbendazol $0.05 \mathrm{ml} \mathrm{kg}^{-1}$ de peso corporal) & $66.95 a$ & $72.50 a$ & $76.39 a$ \\
\hline Error estándar de la media $\left(s_{\bar{x}}\right)$ & 18.91 & 21.74 & 20.80 \\
\hline
\end{tabular}

Letras diferentes en la misma columna indican diferencias estadísticas significativas $(\mathrm{P} \leq 0.05)$.

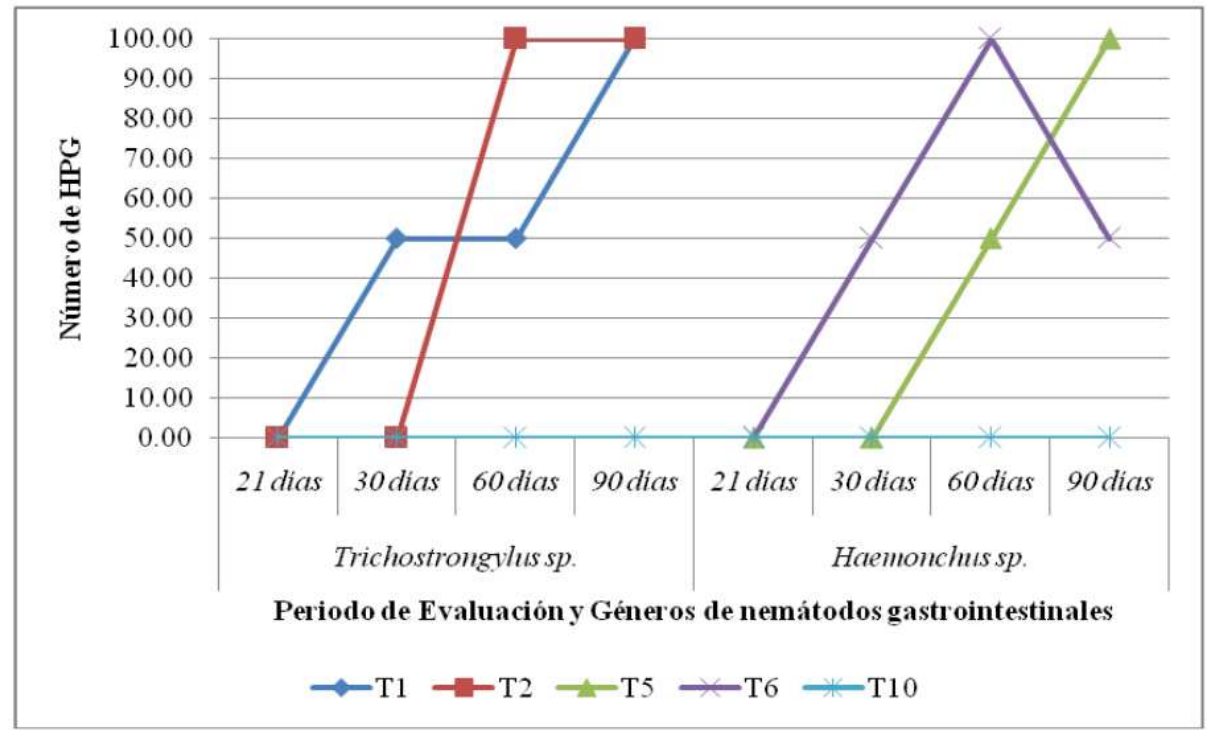

Figura 1. Curva de reinfestación de huevos de nemátodos gastrointestinales a partir de los 21 días post-aplicación en los tratamientos efectivos. Estación Experimental Santa Catalina del INIAP, 2015. 
Zamora, (2014), concluye que la tintura a base de Chenopodium ambrosioides, Cucurbita argyrosperma y Tagetes erecta, administrada por vía oral en bovinos, tiene efecto nematicida, disminuyendo el $55 \%$ de la carga parasitaria de la población evaluada y que los tratamientos a base de la tintura presentaron efecto residual hasta los 28 días posteriores a su aplicación.

\subsection{Peso inicial, peso final y ganancia diaria de peso}

En la Tabla 7 se muestra los promedios de las variables peso inicial, peso final y ganancia diaria de peso. Los promedios de los tratamientos de las variables peso inicial y peso final muestran que los animales seleccionados para este estudio y distribuidos en cada uno de los tratamientos presentaron pesos homogéneos y que los tratamientos en estudio no influenciaron en el peso final. Los promedios de los tratamientos en la variable ganancia diaria de peso permiten señalar que la mayor ganancia se obtuvo en el T10 con 690 gramos; al contrario, el tratamiento T9, presentó la menor ganancia diaria de peso con 500 gramos. En cuanto a los tratamientos en los que se aplicaron los productos naturales, el tratamiento T6 presentó una ganancia diaria de peso de 655 gramos, seguido del tratamiento T2 con 631 gramos.

El promedio de incremento de peso obtenido de $596 \mathrm{~g} \mathrm{día}^{-1}$ está por debajo del parámetro en novillas en crecimiento mencionado por Wattiaux, (2010), que es de $740 \mathrm{~g} \mathrm{día}^{-1}$, pero similar al obtenido por Alfani et al., (1996), con $540 \mathrm{~g} \mathrm{día}^{-1}$ en becerros mestizos lecheros. Por lo señalado, se considera que las respuestas obtenidas están dentro del parámetro normal, debido posiblemente al buen manejo de la nutrición de los animales.

\subsection{Análisis económico de los tratamientos}

Al realizar el análisis de la Tasa de Retorno Marginal (Tabla 8), se encontró que al pasar del tratamiento $\mathrm{T} 9$ al $\mathrm{T} 1$ se obtiene una tasa de retorno marginal de $1,580 \%$, lo que equivale a decir que por cada dólar invertido en el tratamiento T9 para pasar al tratamiento T1 se obtendría un retorno de USD 15.80 dólares; también representa un buen retorno pasar del tratamiento T2 al tratamiento T6, ya que se obtiene una tasa de retorno de $954 \%$, es decir por cada dólar que se invierta al pasar del tratamiento T2 al

Tabla 7. Promedios y error estándar para peso inicial $(\mathrm{kg})$, peso final $(\mathrm{kg})$ y ganancia diaria de peso $(\mathrm{g})$ de los animales durante el periodo de evaluación. Estación Experimental Santa Catalina del INIAP, 2015.

\begin{tabular}{cccc}
\hline Tratamientos en estudio & $\begin{array}{c}\text { Peso } \\
\text { inicial } \\
(\mathrm{kg})\end{array}$ & $\begin{array}{c}\text { Peso } \\
\text { final } \\
(\mathrm{kg})\end{array}$ & $\begin{array}{c}\text { Ganancia } \\
\text { de peso } \\
\text { diaria }(\mathrm{g})\end{array}$ \\
\hline T1= Paico: $0.1 \mathrm{ml} \mathrm{kg}^{-1}$ de peso corporal + una sola aplicación (día 0) & $204 a$ & $217 a$ & $619 a$ \\
T2= Paico: $0.1 \mathrm{ml} \mathrm{kg}^{-1}$ de peso corporal + dos aplicaciones (día 0 y día 1) & $278 a$ & $291 a$ & $631 a$ \\
T3= Paico: $0.2 \mathrm{ml} \mathrm{kg}^{-1}$ de peso corporal + una sola aplicación (día 0) & $275 a$ & $287 a$ & $571 a$ \\
T4= Paico: $0.2 \mathrm{ml} \mathrm{kg}^{-1}$ de peso corporal + dos aplicaciones (día 0 y día 1) & $229 a$ & $242 a$ & $595 a$ \\
T5= Chocho: $0.2 \mathrm{ml} \mathrm{kg}^{-1}$ de peso corporal + una sola aplicación (día 0) & $288 a$ & $301 a$ & $619 a$ \\
T6= Chocho: $0.2 \mathrm{ml} \mathrm{kg}^{-1}$ de peso corporal + dos aplicaciones (día 0 y día 1) & $269 a$ & $283 a$ & $655 a$ \\
T7= Chocho: $0.4 \mathrm{ml} \mathrm{kg}^{-1}$ de peso corporal + una sola aplicación (día 0) & $219 a$ & $230 a$ & $536 a$ \\
T8= Chocho: $0.4 \mathrm{ml} \mathrm{kg}^{-1}$ de peso corporal + dos aplicaciones (día 0 y día 1) & $251 a$ & $262 a$ & $548 a$ \\
T9= Testigo absoluto (sin aplicación de producto) & $322 a$ & $332 a$ & $500 a$ \\
T10= Testigo convencional (Fenbendazol 0.05 ml kg $a$ & $232 a$ & $247 a$ & $690 a$ \\
\hline Error estándar de la media $(s \bar{x})$ & 42.46 & 42.06 & 72.79 \\
\hline Letras diferentes en la misma columna indican diferencias estadísticas significativas (P $\leq 0.05)$. & &
\end{tabular}


Tabla 8. Tasa de Retorno Marginal de los tratamientos no dominados. Estación Experimental Santa Catalina del INIAP, 2015.

\begin{tabular}{|c|c|c|c|c|c|}
\hline Tratamiento & $\begin{array}{l}\text { Total costos } \\
\text { que varían } \\
\text { (USD) }\end{array}$ & $\begin{array}{l}\text { Costos } \\
\text { marginales } \\
\text { (USD) }\end{array}$ & $\begin{array}{c}\text { Beneficios } \\
\text { netos } \\
\text { (USD) }\end{array}$ & $\begin{array}{c}\text { Beneficios netos } \\
\text { marginales } \\
\text { (USD) }\end{array}$ & $\begin{array}{c}\text { Tasa de retorno } \\
\text { marginal } \\
(\%)\end{array}$ \\
\hline $\begin{array}{l}\text { T10= Testigo convencional (Fenbenda- } \\
\text { zol } 0.05 \mathrm{ml} \mathrm{kg}^{-1} \text { de peso corporal) }\end{array}$ & 0.61 & 0.22 & 15.34 & 0.60 & 266 \\
\hline $\begin{array}{l}\text { T6= Chocho: } 0.2 \mathrm{ml} \mathrm{kg}^{-1} \text { de peso cor- } \\
\text { poral + dos aplicaciones (día } 0 \text { y día } 1)\end{array}$ & 0.39 & 0.05 & 14.74 & 0.50 & 954 \\
\hline $\begin{array}{l}\mathrm{T} 2=\text { Paico: } 0.1 \mathrm{ml} \mathrm{kg}^{-1} \text { de peso corpo- } \\
\text { ral + dos aplicaciones (día } 0 \text { y día } 1)\end{array}$ & 0.34 & 0.18 & 14.24 & 0.10 & 58 \\
\hline $\begin{array}{l}\mathrm{T} 1=\text { Paico: } 0.1 \mathrm{ml} \mathrm{kg}^{-1} \text { de peso corpo- } \\
\text { ral + una sola aplicación (día } 0)\end{array}$ & 0.16 & 0.16 & 14.14 & 2.59 & 1,580 \\
\hline $\begin{array}{l}\text { T9= Testigo absoluto (sin aplicación de } \\
\text { producto) }\end{array}$ & 0.00 & & 11.55 & & \\
\hline
\end{tabular}

tratamiento T9 se obtendrá un retorno de USD 9.54 dólares. Estos valores obtenidos son altos y justificarían el empleo de productos orgánicos para el control de parásitos en animales jóvenes.

El análisis económico de presupuesto parcial se basó en los costos de los insumos y su preparación; un litro de extracto acuoso de paico cuesta USD 0.67 dólares y un litro de extracto fitoquímico del paico USD 0.83 dólares, en cambio, un litro de Febendazole tiene un costo de USD 40 dólares. El precio referencial de un kilogramo de carne de un animal en pie fue de USD 1.10 dólares, tomando en cuenta los mercados de Machachi, Quito y Santo Domingo de los Tsáchilas.

\section{Conclusiones}

Los parásitos identificados en los animales en estudio fueron los nematodos Trichostrongylus sp. y Haemonchus sp., y los protozoarios del género Eimeria sp. El efecto de la aplicación de los productos evaluados sobre la carga parasitaria de los géneros de parásitos gastrointestinales presentes en bovinos jóvenes fue diferente en términos de ingredientes activos, dosis, frecuencias de aplicación y período de evaluación. El tratamiento T10 fue efectivo en el control de los géneros Trichostrongylus sp. y Haemonchus sp. en todo el período de evaluación. Los tratamientos T1 y T2 fueron efectivos para el control del género Trichostrongylus sp.; los tratamientos T5 y T6 fueron efectivos para el control de Haemonchus sp.; y los tratamientos T2 y T5 redujeron la carga parasitaria del género Eimeria sp. No se presentó reinfestación en el tratamiento T10, mientras que en los tratamientos orgánicos la reinfestación se dio alrededor de los 30 días después del período de evaluación. El porcentaje de efectividad de los tratamientos no influyó significativamente en la ganancia diaria de peso. Los tratamientos efectivos con los productos orgánicos son una alternativa económicamente viable para el control de parásitos gastrointestinales en bovinos jóvenes.

\section{Agradecimientos}

Al Programa de Ganadería de la Estación Experimental Santa Catalina del INIAP, por prestar la infraestructura, semovientes, equipos, etc. para la realización de este estudio. Al Laboratorio de Diagnóstico Clínico Veterinario "VETELAB" por realizar los análisis de laboratorio.

\section{Bibliografía}

Alfani, G., M. Ventura, D. Esparza, D. Dean y A. del Villar. 1996. Evaluación de diferentes sistemas de alimentación en becerros mestizos lecheros. Rev. Fac. Agron. (LUZ), 13:115-134. 
Álvarez, C., P. Rodríguez y E. Carvajal. 2011. Efecto del extracto de paico (Chenopodium ambrosioides), en parásitos gastrointestinales de gallos de pelea. Fundación Universitaria Juan de Castellanos. Revista Cultura Científica JDC. páginas 76-80.

Anziani, O. y C. Fiel. 2004. Resistencia de los nemátodos gastrointestinales a los antihelmínticos: un problema emergente y relevante para la producción bovina nacional. Resistencia a los antiparasitarios internos en Argentina, FAO, Producción y Sanidad Animal. páginas 40-49.

Angulo, F. 2005. Nematodosis gastrointestinales. Manual de ganadería doble propósito. Cátedra de Enfermedades Parasitarias, Facultad de Ciencias Veterinarias, Universidad del Zulia. Maracaibo, Venezuela. páginas 377-383.

Borchert, A. 1993. Parasitología Veterinaria, $3^{r a}$ Ed. Zaragoza, España. Editorial Acribia. páginas 24-28.

Caracostántogolo, J., M. Peña, J. Schapiro, C. Cutullé, R. Castaño y G. Balbiani. 2005. Manejo de Parásitos Internos en los Bovinos. http://www.biblioteca.org.ar/libros/210275. pdf. Consultado 22 de octubre de 2014.

Castro, J., M. González y M. Mezo. 2011. Principales parasitosis en el ganado vacuno lechero: pautas racionales de control. http://www.ciam.es/pdf/Parasitoloxia.pdf. Consultado 3 de febrero del 2015.

Chacón, E., P. Espinoza, M. Sánchez y O. Vásquez. 2009. Aplicación del extracto del paico en la eliminación de parásitos intestinales en niños y niñas del tercer grado " $\mathrm{C}$ " de primaria, de la Institución Educativa Nacional Julio Gutiérrez Solari del Municipio El Milagro. Trujillo, Perú. Escuela de Enfermería. Escuela de Ciencias Médicas. páginas 35-42.

CIMMYT. 1988. La formulación de recomendaciones a partir de datos agronómicos: Un manual metodológico de evaluación económica. D.F. México.

Cordero, M., F. Rojo y A. Martínez. 2005. Parasitología Veterinaria. La reimpresión $1^{\text {ra }}$ Ed. Madrid, España. Editorial McGraw Hill Interamericana. páginas 519-546.
Díaz, E. 2013. Principales parásitos externos e internos de los animales domésticos. Atlas de parasitología veterinaria. http://www.slideshare.net/Parasitismo/atla s-de-parasitologia-veterinaria. Consultado 20 de enero de 2015.

Estrada-Celi, G., D. Castaño-Piamba, K. Ramírez Arango, J. Rodríguez Quintero y L. González Montilla. 2012. Estudio de la eficacia del paico (Chenopodium ambrosioides) como antihelmíntico en especímenes silvestres mantenidos en cautiverio en el Hogar de Paso de Fauna Silvestre de la Universidad de la Amazonía. CES Medicina Veterinaria y Zootecnia. 7(2):31-36.

FAO. 2003. Resistencia a los antiparasitarios, estado actual con énfasis en América Latina. Food and Agriculture Organization of the United Nations. página 51 .

Fiel, C., P. Steffan y D. Ferreyra. 2011. Diagnóstico de las parasitosis más frecuentes de los rumiantes: técnicas de diagnóstico e interpretación de resultados, $1^{\text {ra }}$ Ed. Buenos Aires, Argentina. Editorial Tandil. páginas 14-26.

Fierro, M. 2010. Diagnóstico parasitario, evaluación de la eficiencia antihelmíntica y diseño de un plan sanitario parasitológico en la caravana de alpacas de la comunidad Morochos, cantón Cotacachi. Tesis de Grado. Facultad de Ciencias Pecuarias, Escuela de Ingeniería Zootécnica, Escuela Superior Politécnica de Chimborazo. Riobamba, Ecuador. páginas 60-61.

Finkeros. 2012. Uso de paico como antiparasitario. http:/ /abc.finkeros.com/uso-de-paico-como -antiparasitario/. Consultado 16 de enero del 2015.

González, R., G. Torres, N. Nuncio, J. Cuéllar y M. Zermeño. 2003. Detección de eficiencia antihelmíntica en nemátodos de ovinos de pelo con la prueba de reducción de huevos en heces. Investigación Ganadera de Desarrollo Rural $\mathbf{N}^{\circ}$ 15. página 12.

Gross, R. 1992. El cultivo y la utilización del Tarwi (Lupinus mutabilis Sweet). Estudio FAO. Editorial GTZ. páginas 141-169.

INAMHI. 2013. Anuario meteorológico. Instituto Nacional de Meteorología e Hidrología. Estación Meteorológica Izobamba. Mejía-Ecuador. 
James Brown Pharma. 2013. Ficha técnica del producto Bendacur, antiparasitario oral. F-07. http://www.jamesbrownpharma.com/image s/stories/ParaWeb/Bendacur.pdf. Consultado 4 de marzo del 2015.

Jurado, C., J. Duarte y O. López. 2007. Recuperación de los conocimientos tradicionales relacionados con la salud de bovinos a pequeña escala en Villamaría. Caldas, Colombia.

Maya, A. y J. Quijije. 2011. Determinación de la carga parasitaria en tres especies zootécnicas (Bos taurus, Ovis aries y Equus caballus) y su relación con las condiciones, Ecuador. página 1.

Miño, L., B. Pila y M. Sacoto. 1998. Diagnóstico parasitario en bovinos del IASA y su control mediante la aplicación de tres antiparasitarios con diferente principio activo. Tesis de Ingeniería. Carrera de Ciencias Agropecuarias I.A.S.A.1. Sangolquí, Ecuador. páginas 15-30.

Morales, G., L. Pino, E. Sandoval, J. Florio y D. Jiménez. 2006. Niveles de infestación parasitaria, condición corporal y valores de hematocrito en bovinos resistentes, resilientes y acumuladores de parásitos en un rebaño Criollo Río Limón. Zootecnia Trop. 24(3):333-346.

Morales, G., L. Pino, L. González y C. Balestrini. 2003. Efecto de la carga parasitaria y del número de especies de Strongylida sobre el recuento de huevos por gramo en bovinos naturalmente infectados. Veterinaria Tropical. 28:8.

Muñoz, J. 2013. Homeopatía en rodeos productivos. http://www.homeovet.com.ar/producci on/Homeopatia_en_rodeos_productivos.html. Consultado 30 de enero de 2015.

OIE. (2005). Reglamento técnico pruebas de eficacia para registro de antiparasitarios internos para rumiantes. XI seminario sobre armonización del registro y control de medicamentos veterinarios. Montevideo, República Oriental del Uruguay. páginas 8, 9, 13 .

Prada, G. 2008. Determinación de las características morfológicas de larvas L1, L2 y L3 en parásitos gastrointestinales del equino en la región de Los Lagos, Chile. Revista de Medicina Veterinaria. 15:39-48.
Power, K., I. Wood, J. Eckert, T. Gibson y H. Smith. 1982. Directrices para evaluar la eficacia de los antihelmínticos en rumiantes (bovinos y ovinos). Vet. Parasitología. páinas 265-284.

Sixtos, C. 2011. Procedimientos y técnicas para la realización de estudios coproparasitoscópicos. Virbac al día. Publicación trimestral de actualización científica y tecnológica $\mathbf{N}^{\circ} \mathbf{2 4}$. México. páginas 6-9.

Soca, M., E. Roque y M. Soca. 2005. Epizootiología de los nemátodos gastrointestinales de los bovinos jóvenes. Red de Revistas Científicas de América Latina, Caribe, España y Portugal. Estación Experimental de Pastos y Forrajes "Indio Hatuey". Cuba. 28(3):177-178.

Supe, C. 2008. Utilización de plantas desparasitantes tradicionales: paico, ajenjo, ruda y marco en el control de parásitos gastrointestinales en cuyes. Tesis de grado. Facultad de Ciencias Pecuarias, Escuela de Ingeniería Zootécnica, Escuela Superior Politécnica de Chimborazo. Riobamba, Ecuador. página 22.

Thienpont, D. 1989. Diagnóstico de la Helmintiasis por medio del examen coprológico, $2^{\text {da }} \mathrm{Ed}$. Reerse, Bélgica. Editorial Janssen Foundation. páginas 80-85.

Torres, L. 2006. Utilización del chocho (Lupinus mutabilis Sweet) como antiparasitario gastrointestinal y hepático en ovinos mestizos. Tesis de grado. Facultad de Ciencias Pecuarias, Escuela de Ingeniería Zootécnica, Escuela Superior Politécnica de Chimborazo. Riobamba, Ecuador. páginas 37-38.

Trujillo, S. y B. Madrigal. 2005. Plantas antimaláricas de Tumaco, Costa Pacífica, Colombia. Editorial Universidad de Antioquia. Tumaco, Colombia. página 125 .

Valencia, C. 2014. Consideraciones para la elaboración de un calendario de desparasitación en ganado bovino. http://web.altagenetics.com/ecuador/Dairy Basics/ Details/9171_Consideraciones-para -la-elaboracion-de-un-calendario-de-despara sitacion-en-ganado-bovino.html. Consulta: 28 de octubre de 2015. 
Wattiaux, M. 2010. Crianza de novillas del destete al parto, capítulo 34: tasa de crecimiento. Instituto Babcock para la Investigación y Desarrollo Internacional de la Industria Lechera. Universidad de Wisconsin, Madison. página 133.
Zamora, L. 2014. Comparación del efecto nematicida de tres diferentes tratamientos en bovinos. Universidad de San Carlos de Guatemala. Tesis Doctoral. Guatemala. 